\title{
DESIGN AND PARAMETRIC INVESTIGATIONS OF PERMANENT MAGNET ADHESION MECHANISM FOR ROBOTS CLIMBING ON REINFORCED CONCRETE WALLS
}

\author{
VIJAYA GOPALA RAO M.V., ADITHYA BALACHANDRAN, SHEETAL P JADHAV, \\ TULA SRIDATH, MANJU MOHAN, RM. KUPPAN CHETTY, D. DINAKARAN, M.M. RAMYA \\ Centre For Automation and Robotics, School of Mechanical Sciences \\ Hindustan Institute of Technology and Science, Chennai-603103, India \\ E-mail:kuppanc@hindustanuniv.ac.in \\ www.hindustanuniv.ac.in \\ MOHAMMAD O. TOKHI AND TARIQ P. SATTAR \\ School of Engineering, London South Bank University, United Kingdom
}

\begin{abstract}
Wall Climbing Robots (WCRs) have found extensive applications in the past decade in numerous engineering fields, however, the design of efficient adhesion mechanism for robots climbing on concrete surfaces remains a challenge and attracts research attention. This paper proposes various designs of magnetic adhesion mechanism for concrete surfaces and investigates the adhesion force and payload capacities each design would accommodate for wall climbing robot applications. Permanent magnet is used as the magnetic adhesion mechanism and a yoke structure helps in holding the magnets and influences the adhesion characteristics of the mechanism. The effect of various structural designs of adhesion mechanisms on the adhesion force and payload capacity on the concrete surface is studied in this work. The adhesion forces against the different standoff distances which comprise the gap between the magnet and the concrete surface are also investigated therein. The results show that the developed adhesion mechanism can be applied for concrete walls generating the required adhesion forces and providing a better insight in choosing the best configuration, number of magnets and standoff distances for the design of adhesion mechanism against the required payload of WCR.
\end{abstract}

Keywords: Adhesion mechanism; Wall climbing robot; Permanent magnets; Neodymium; magnetic.

\section{Introduction}

The growth in civilization and the thirst of human to urbanize his standard of living has led to the construction of luxurious and sky-high tall buildings. But, with the increased number of buildings and the advancements in architectural designs, the responsibility for humans to provide regular maintenance and inspections of these buildings has also increased in order to sustain the beauty of the buildings. In the earlier day's inspection of cracks or defects on the wall columns, dam structures, high-rise buildings, etc. were a matter of high risk, as human need to climb and inspect the structures. Although, the availability of equipment for enabling a person to perform inspection on the buildings is abundant, the risk factors could not be reduced as humans are prone to having panic attacks or vertigo sensations or other unforeseen effects which could harm or kill a person during climbing the buildings or structures for inspection/maintenance works. Thanks to technological developments that now humans directly/physically need not be involved in the inspection/maintenance works of the buildings as gadgets like drones and wall climbing robots are available for inspection. The usage of robotics for the process automation task such as automation of inspection has not only reduced the risk factor involved in terms of human life but also according to Gartner's hype cycle, the 
forecasted market revenue for robotics in the year 2019 was around \$13 billion. Thus, helping in terms of economic factors too.

There are various adhesion mechanisms such as vacuum suction, magnetic attraction etc, that can be employed for a robot to adhere to the surface of the wall. But the best and the most reliable mechanism is the magnetic adhesion mechanism as this mechanism ensures proper hold without losing the grip of the robot with respect to the surface it adheres. The proper design of the magnets and their placements yield optimal results in terms of efficient adhering forces between the wall surface and the robot. Various existing adhesion mechanisms and wall climbing robots are analyzed such as the one by Yanagimura et al. [1], who propose a method by which an MAV (Micro Air Vehicle) can travel in air without any involvement of propulsion systems. For this to achieve, they designed and developed a magnetic adhesion system which consists of a switch, a winch with servomotor and a rope/cable, a shaft with spring coiled around and a magnet attached to the end of the shaft. Ward et.al, [2] have presented a design for the electro permanent magnetic $[\mathrm{EPM}]$ adhesion device for optimal holding power of the magnets on the metal surfaces with less weight of the adhesion device and simultaneously provide an efficient failsafe solution to the robot involved in the climbing process. Berengueres et al. [3] have proposed a distributed compliant device for holding/gripping purposes which is inspired by a gecko foot mechanism.

Lee et al. [4] have proposed a non-contact magnetic adhesion system which enables a robot to move around the surface and, efficiently pass over obstacles on the surface, while holding the robot's position intact in any orientation. Li et al. [5] have proposed a new wall climbing robot mechanism for easy wall-to-wall transition and for high payload requirements. They named their robot as 'combot'. Their robot can carry a payload of about $10 \mathrm{~kg}$, and it is applicable to heavy industrial applications. Sekhar et al. [6] have proposed a method to develop a low cost and efficient wall climbing robot that can climb on almost all surfaces to ensure mechanical stability. Howlader et al. $[7,8]$ have proposed a method for wall climbing robot by changing the mechanical design parameters such as the magnet orientation, the distance between the magnets, and yoke thickness using finite element analysis.

Wile et al. [9] have proposed and created a miniaturized wall climbing robot with smart robotic feet using suction pumps that can climb from floor to wall. It uses pic microcontroller and 6-volt battery as the power source. Yehy et al. [10] have proposed a method to create wall climbing robot using unipolar electro adhesion pad of aluminium and copper. The electrostatic adhesion is produced by electrostatic field, but the force created is not great compared to the magnetic field. Silva et al. [11] have proposed a model of a wall climbing robot using the Bernoulli principle of suction vacuum pumps on concrete walls. Howlader et al. [8] have aimed to develop a unique adhesion mechanism for wall climbing robot to automate the NDT inspection of large critical reinforced concrete structures.

Kanjanapan et al. [12] have proposed a model of a robot that can climb a surface and has the ability to move back to the climbing surface when the robot starts to fall out of the wall. Tovarnov et al. [13] have developed a model of a magnetic-tape locomotion mechanism for wall climbing robot and have proposed mathematical model of the motion of a mobile robot on vertical surface. Shen et al. [14] have described the design aspects of locomotion and magnetic adhesion of permanent magnet and its embedded system. Wei et al. [15] proposed a lightweight magnetic system to provide an optimum adhesion force and at required tire pressure to maintain a specified air gap between the magnets and the surface for a wall climbing robot. Jose et al. [16] have proposed the necessary conditions required for a wall climbing robot such as the locomotion and adhesion techniques. Nansai et al.[17] have discussed various requirements for cleaning wall climbing robot and categorized into 6 classes based on the adhesion mechanism. 
Sattar et al. [18] have proposed different methods to optimize the magnetic adhesion for ferrous surface. Hussain et al. [19] have designed a robot framework for magnetic adhesion for ferromagnetic surface using ANSYS FEM software for an efficient design.

Based on the literature survey of the above papers, it is understood that wall climbing robots, or the adhesion mechanisms used for enabling the robot to adhere the wall surface are not stable enough to attract a concrete wall column. Also, the magnetic adhesion methods used are only useful for adhering the robot to ferromagnetic surfaces or metal bodies. Therefore, this research focuses on addressing the above stated issues and thus designing magnetic adhesion mechanisms for attracting concrete wall columns by attracting the steel rebars inside the concrete wall column. The work focuses more on the properties of the magnet and studies how their orientation can influence the magnetic attraction. The N52 grade magnet is chosen as it is the highest grade of magnet available in the market and has high penetrating magnetic flux which caters for the intended purpose.

\section{Materials and Methodology}

The work presented in this paper focuses on the magnetic adhesion and the payload capacity of a magnetic adhesion mechanism for various adhesion designs. The three-dimensional models of the adhesion mechanisms and the concrete wall column are developed using SOLIDWORKS CAD software. The simulation studies are carried out using ANSYS MAXWELL 3D software.

\subsection{Materials Used}

The materials used in the simulations comprise concrete wall column, neodymium permanent magnets (NdFeB), and yoke (Electric Steel, Perm Alloy, Iron, Ferrite, Cobalt Iron). The concrete wall column comprises of steel rebars, which are enclosed in a cement concrete structure with adequate plastering. Although, there is a variation in the distance between the internal steel rebar and the external concrete covering, for this work, the distance between the steel rebar and the concrete covering inclusive of plastering is $30 \mathrm{~mm}$. The properties of the steel rebar used for the simulation are presented in Table 1.

Table 1: Properties of steel rebar

\begin{tabular}{cc}
\hline Properties & Value \\
\hline Bulk conductivity & 2000000 siemens $/ \mathrm{m}$ \\
Young's modulus & $2 \times 1010 \mathrm{~N} / \mathrm{m} 2$ \\
Poisson's ratio & 0.25 \\
Relative permeability of steel rebar $(\mu \mathrm{r})$ & 1500 \\
\hline
\end{tabular}

The type of permanent magnet used in this work is NdFe52 (Neodymium Ferrous Boron). This magnet is made of an alloy which primarily consists of Neodymium, Boron, and Iron. Compared to other permanent magnets available such as Alnico, Ferrite and Samarium cobalt, the neodymium magnets are the most powerful magnets. These magnets are available in various sizes and shapes. Different size of magnets would also influence the payload capacity. The properties of the N52 magnet are presented in Table 2. Adding yolk to the design of the adhesion mechanism increases the magnetic flux, therefore influencing the payload capacity. This is due to the magnetic coupling of the yolk with magnets. Optimizing the arrangement of the magnets with the help of yoke would also influence the payload capacity. The material used for the yoke should be highly permeable in nature in order to aid in increasing the magnetic flux density. The derivatives of iron such as electrical steel, cobalt iron, perm alloy, iron and ferrite are found to be highly permeable and are considered as yoke materials for this study. Table 3 presents the properties of electrical steel, perm alloy, iron, ferrite, and cobalt iron, respectively. 
Table 2. Properties of NdFe52 permanent magnet

\begin{tabular}{cc}
\hline Properties & Value \\
\hline Magnetic induction intensity $\mathrm{Br}(\mathrm{T})$ & 1.31 \\
Coercive force $\mathrm{Hcb}(\mathrm{KA} / \mathrm{m})$ & 915 \\
Intrinsic coercive force $\mathrm{Hci}(\mathrm{KA} / \mathrm{m})$ & 955 \\
Magnetic energy product $\mathrm{HB}(\mathrm{KJ} / \mathrm{m} 3)$ & 318 \\
Relative permeability $(\mu \mathrm{r})$ & $1.068-1.113$ \\
Relative permeability of yoke $(\mu \mathrm{r})$ & 5000 \\
Relative permeability of steel concrete surface $(\mu \mathrm{r})$ & 1 \\
Relative permeability of steel rebar $(\mu \mathrm{r})$ & 1500 \\
\hline
\end{tabular}

Table 3 Properties of yoke materials in ANSYS

\begin{tabular}{cccccc}
\hline Properties & Electric Steel & Perm-alloy & Iron & Ferrite & Cobalt Iron \\
\hline Relative permeability & 4000 & 9000 & 5000 & 1000 & 1800 \\
Young's modulus (GPa) & 190 & 113 & 195 & 119 & 230 \\
Poisson's ratio & 0.24 & 0.25 & 0.28 & 0.2 & 0.28 \\
\hline
\end{tabular}

\section{Simulation Studies}

\subsection{D model of the adhesion mechanism and the concrete wall column}

The development of 3D models for the adhesion mechanisms and the concrete wall column is carried out using SOLIDWORKS software. The following steps are followed:

a) Problem definition: The problem begins with specifying the dimensions, the face/axis of the $2 \mathrm{D}$ drawing being made, the required $3 \mathrm{D}$ extrude/cut to be performed, and finally, saving the file in STEP format for the simulations to be carried out in ANSYS MAXWELL 3D software.

b) Drawing the model: All the dimensions are set in millimeter $(\mathrm{mm})$. The model of the adhesion mechanism/concrete wall column begins by selecting the face/view (Top/Side/Front Views) on which the blueprint of the 3D model is to be made.

c) Performing Extrude/Cut Operations: After the blueprints of the necessary 3D models of the adhesion mechanism, the concrete wall column is made. the specific entities such as the magnet, internal steel rebars, external concrete, square brackets and the yoke material are either extruded/intruded/cut as per the necessary requirements and dimensions. The concrete column is of cross section $300 \mathrm{~mm}$. These measurements for the concrete column are considered as per standards followed in general constructions.

d) Saving File and Exporting: The developed 3D models are assembled using the "Align Object" tool as shown in Figures 1 and 2. The adhesion mechanism and the concrete wall column are attached to each other in a similar way as stated above, and this is depicted in Figure 3. The assembled files are saved with ".step" extension. The STEP extension is required as SOLIDWORKS is a proprietary software and has ".sldprt"/“.sldasm” extension. In order to export the developed 3D model to ANSYS MAXWELL 3D for further simulation the "step" extension acts as an aid to bridge the gap between the two proprietary softwares i.e., "SOLIDWORKS" and "ANSYS MAXWELL 3D". 


\subsection{Simulation Using ANSYS MAXWELL 3D}

ANSYS Maxwell 3D was used to find the adhesion force on the steel rebars. The focus is to demonstrate the forces acting on the internal steel rebar and payload the magnets can carry, and this software has the features necessary for this work. ANSYS has a built-in library so that the required materials for the magnets, yoke (electric steel, perm alloy, iron, ferrite and cobalt iron), wall column, and steel rebars could be defined in the software. In order to simulate the 3D model, a specific space must be defined and hence a boundary is created around the 3D model. The material for the boundary enclosure is selected as air (as air is the natural media between the magnets and the concrete wall column). The following simulations are performed in ANSYS MAXWELL 3D.

\section{a) 5 Magnets of various dimensions in $X$ shape yoke structure}

The ' $X$ ' shape yoke configuration as shown in Figure 1 was simulated with various sizes of magnet with $13 \mathrm{~mm}$ thickness. The distances between the concrete wall and the magnets are considered as $2 \mathrm{~mm}, 4 \mathrm{~mm}, 6 \mathrm{~mm}$, and $10 \mathrm{~mm}$. The yoke thickness is assigned as $10 \mathrm{~mm}$. The simulations are performed on the $\mathrm{X}$-shaped yoke and rectangular shaped yoke by varying the dimensions of the magnets and varying the number of magnets and keeping the yoke material as iron and assigning the distance between the wall and the magnets as $2 \mathrm{~mm}$ constant. These results are tabulated in Table 4. This proves that increase in magnet size can increase the overall magnetic force/payload capacity. Figure 3 depict the ANSYS simulation environment with the respective magnetic flux lines passing through the steel rebars.

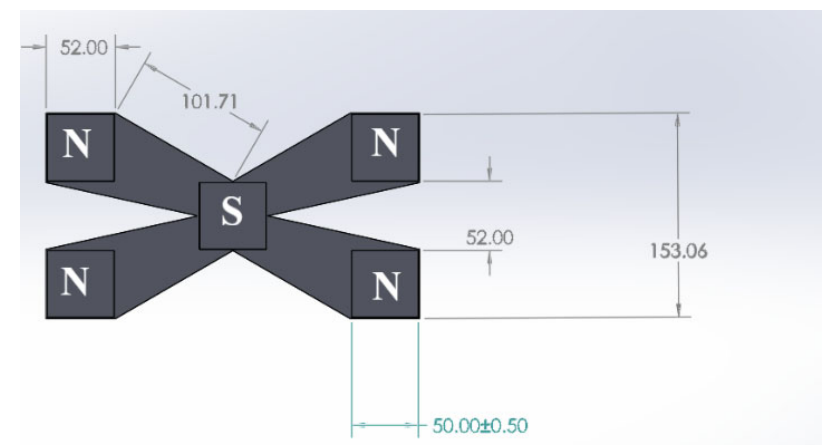

All Dimensions in mm.

Figure1. 'X' shaped yoke with magnets of different thicknesses using SOLIDWORKS

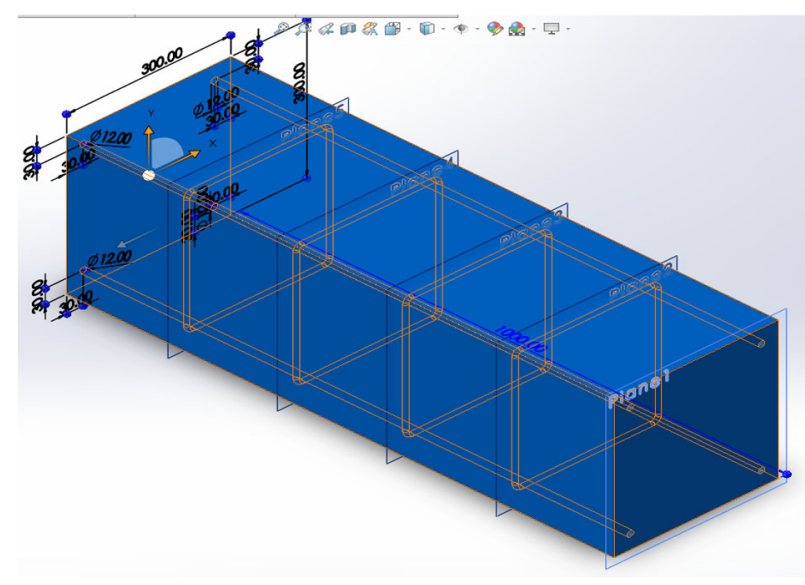

Figure 2. Concrete Cement block with square brackets 


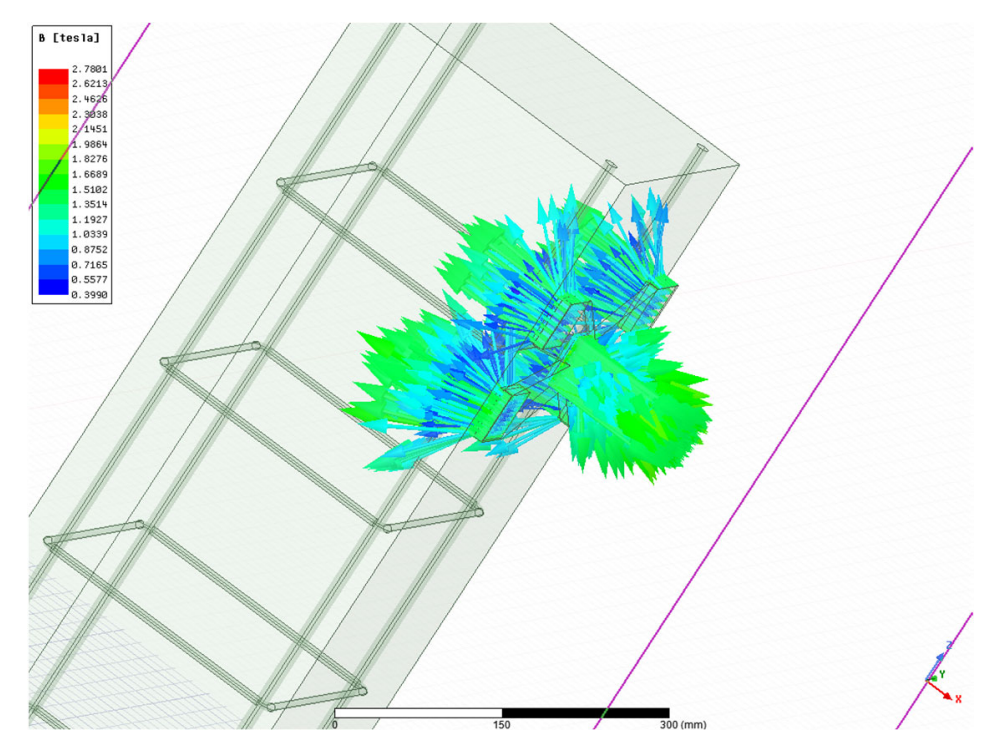

Figure 3. Magnetic flux line generated using ANSYS simulation

Table 4. Results for NdFe52 magnet with varying dimensions.

\begin{tabular}{ccccccc}
\hline Magnets & $\begin{array}{c}\text { Length, } \\
\text { width, } \\
\text { thickness in } \\
(\mathrm{mm})\end{array}$ & $\begin{array}{c}\text { Yoke } \\
\text { material }\end{array}$ & $\begin{array}{c}\text { Number of } \\
\text { Magnets }\end{array}$ & Yoke shape & $\begin{array}{c}\text { Distance } \\
\text { from the } \\
\text { concrete wall } \\
\text { in (mm) }\end{array}$ & $\begin{array}{c}\text { Force } \\
(\mathrm{N})\end{array}$ \\
\hline Ndfe52 & $30,30,13$ & Iron & 5 & X shape & 2 & 3 \\
Ndfe52 & $40,40,13$ & Iron & 5 & X shape & 2 & 8.33 \\
Ndfe52 & $50,50,13$ & Iron & 5 & X shape & 2 & 18.56 \\
Ndfe52 & $50,50,13$ & Iron & 4 & Rectangle & 2 & 31.40 \\
\hline
\end{tabular}

According to Table 4, the magnet of the size $50 \mathrm{~mm} \times 50 \mathrm{~mm} \times 13 \mathrm{~mm}$ of rectangular yoke shape has the highest magnet adhesion force among the rest. This proves that increase in the magnet size can increase the overall magnetic force.

\section{b) 3 and 6 magnets at equal distances in rectangular shape yoke}

The 3-magnet configuration consists of magnets of length and width $50 \mathrm{~mm}$ and depth $13 \mathrm{~mm}$. In this case, the yoke material has been assigned as ferrite and iron, the dimensions of magnets are kept constant, instead the distance is varied between the wall column and the magnets as in [18-19]. The simulation results for the adhesion forces at various standoff distance for different yoke materials is shown in Figures 4 (a) and (b). From the results it is understood that an increase in the number of magnets would increase the adhesion force at the respective standoff distances

Similarly, the simulations are carried out for 6 magnet configurations placed at equidistance as in [18-19]. Different yolk materials were assigned such as iron, ferrite, electric steel, perm alloy and cobalt iron and checked whether the yolk material can improve the payload capacity. In this simulation the distances between the wall column and magnets i.e. standoff distance are also varied. Figure 5 depict the simulations results for the configurations obtained. It could be observed that, the 6-magnet configuration provides less adhesion force as compared to other configurations as observed in Figure 4. This is because of the discrepancy/noncompliancy between the placement and alignment of the rectangular adhesion mechanism and the steel rebars inside the concrete wall column. 


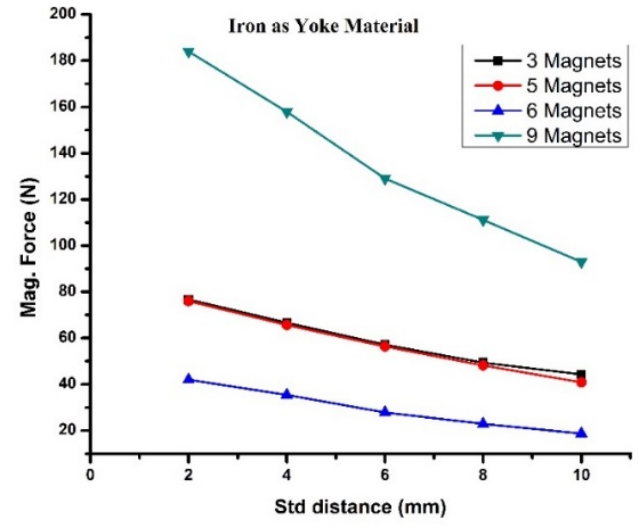

(a)

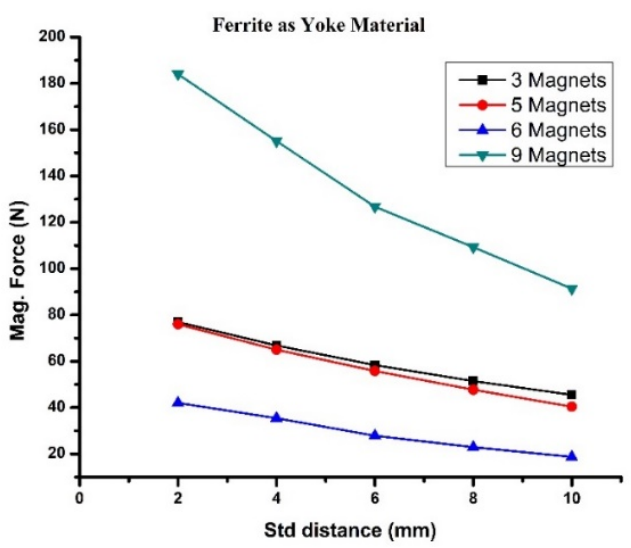

(b)

Figure 4 (a). Comparison between different yoke configuration using iron as a yoke material (b). ferrite as a yoke material
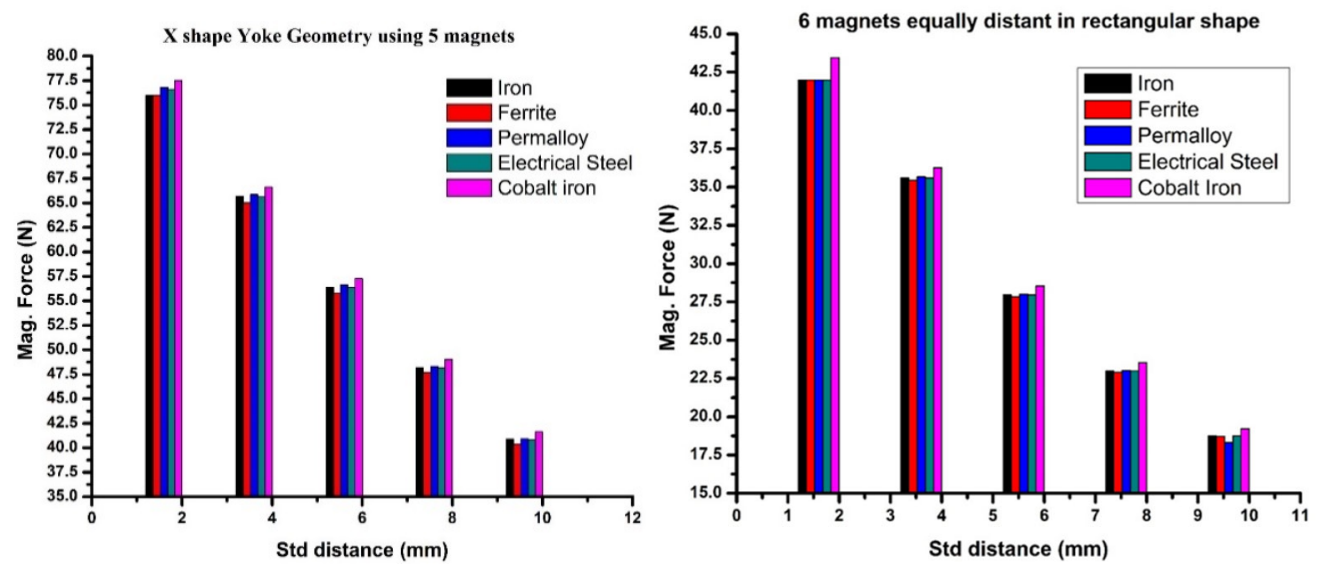

Figure 5. Comparison between different of adhesion force against the standoff distance for different yoke configuration and yoke material, respectively. (a) for X- shape with 5 magnets (b) Rectangular shape with 6 magnets

Figure 5 depicts the values of payload capacities obtained while using different yoke materials and various standoff distances such as $2 \mathrm{~mm}, 4 \mathrm{~mm}, 6 \mathrm{~mm}$, and $10 \mathrm{~mm}$. It is evident from Figure 5, irrespective of the materials, adhesion mechanism yields a maximum adhesive force when the standoff distance i.e. the distance between the wall and the adhesion mechanism of the WCR is at minimum level. For all the yoke materials, the maximum adhesive force obtained is when standoff distance is at $2 \mathrm{~mm}$. Thus, The results obtained through this work, shows that the developed adhesion mechanism can be applied for concrete walls to generate the required adhesion forces and provide a better insight in choosing the best configuration, number of magnets and standoff distances for the design of adhesion mechanism against the required payload of WCR.

\section{Conclusion}

In this study, an adhesion mechanism using permanent magnets has been developed for robots climbing on reinforced concrete walls. The magnetic flux density describing the payload capacity is visualized and analyzed using the simulations. The effect of various structural designs of adhesion mechanisms on the adhesion force and payload capacity on the concrete surface reinforced with metal rods is also studied in this work. The significance of yoke design, yoke material selection and size of the magnet and the vital role they play in determining the 
magnetic force/payload capacity is also explored. Through this research work, it is understood that out of all adhesion mechanisms, the "X-Shaped" yoke provides an optimum payload hold of $7.6 \mathrm{~kg}$ with almost any of the specified yoke material used and the specified magnet size. During the study, out of all the composites of iron mentioned above, ferrite/iron material is most commonly available in the market and is economically a feasible option compared to other composites of ferrite/iron. Although, the ferrite material is heavier in terms of weight, the specified "X-Shape" design helps in removing the extra bulk out of the total material and also would help to yield optimum result and efficiency in terms of overall weight consideration when this magnetic adhesion mechanism is used as part of a wall climbing robot development. Moreover, based on the payload requirements, the required number of magnets can be chosen for the adhesion mechanism. This leaves space for further investigation and the methodology used in this paper could be extended to build a wall climbing robot with magnetic adhesion mechanism.

\section{Acknowledgments}

Authors would like to thank the Royal Academy of Engineering, UK for supporting this research work under Industry-Academia Partnership Programme, Newton funding, the project numbered - IAPP18-19\27

\section{References}

1. K. Yanagimura, K. Ohno, Y. Okada, E. Takeuchi and S. Tadokoro S. IEEE Int. Conf. on Robotics and Automation (ICRA), 6250 (2014)

2. P. Ward and D. Liu. IEEE Int. Conf. on Robotics and Biomimetics (ROBIO), 217 (2012)

3. J. Berengueres, K. Tadakuma, T. Kamoi and R. Kratz. Int. Conf. on Robotics and Automation (ICRA), 1256 (2007)

4. G. Lee, G. Wu, J. Kim and T. Seo. Robotics and Autonomous Systems. 60 (10), 1308 (2012)

5. J. Li, X. Gao, N. Fan, K. Li and Z. Jiang. Int. Conf. on Mechatronics and Automation. (2009)

6. S.R. Aravind, A. Mary, N.R. Sanju, G.R. Akhil, S. Vijitha and B. Gauri. $4^{\text {th }}$ Int. Conf. on Intelligent Systems, Modelling and Simulation, (2013)

7. M.O. F Howlader. Development of a Wall Climbing Robot and Ground Penetrating Radar System for Non-Destructive Testing of Vertical Safety Critical Concrete Structures. (2016).

8. M.O.F. Howlader and T. Sattar. Int. J. of Adv. Comp. Sc. and App. 6(8), 8 (2015)

9. G. Wile, D. M. Aslam. Int. Conf. on Cybern. and Inf. Technol., Syst., and Appl. (CITSA). 87 (2007)

10. M.I. Yehya, H. Salman, W. Ahmad, J. Mirza and H. Abdalla. Int. J. of Robotics and Mechatronics, 2(1), 1 (2016)

11. M.F. Silva, S.B. Ramiro, and A.L.C. Oliveira. J. of Robotics. 906545, (2012)

12. S. Kanjanapan, M. Pruttapon and S Konlayut. IEEE Int. Conf. on Robotics and Biomimetics (ROBIO). (2017)

13. M.S. Tovarnov and N.V. Bykov. J. of Mach. Man. and Reliab. 48(3), 250 (2019)

14. W. Shen, J. Gu and Y. Shen. IEEE Int. Conf. Mechatronics and Automation. (2005)

15. S. Wei, J. Hongjian, W. Tao, J. Daxiong and Z. Shiqiang. Adv. in Mech. Engg. 10(7), (2018)

16. J. Jose, D. Dinakaran, M.M. Ramya and D.G. Harris. Int. J. of Mech., and Prod. Engg. Research and Development. 8(6), 59 (2018)

17. S. Nansai and R. Mohan. Robotics. 5(3), 14 (2016)

18. T. Sattar, H. Salman and E. Salinas. Int. Conf. on CAD/CAM, Robotics and Factories of the Future. (2011)

19. S. Hussain, T.P. Sattar and E. Salinas. Int. J. of Intel. Sys. Tech. and Appl. 11(1-2), 102 (2012) 